\title{
Mechanisms of Azole Resistance and Trailing in Candida tropicalis Bloodstream Isolates
}

\author{
Pao-Yu Chen ${ }^{1,2}$, Yu-Chung Chuang ${ }^{1}$, Un-In Wu ${ }^{1}$, Hsin-Yun Sun ${ }^{1}$, Jann-Tay Wang ${ }^{1}$, Wang-Huei Sheng ${ }^{1,3}$, \\ Yee-Chun Chen $1,3,4, *$ (i) and Shan-Chwen Chang ${ }^{1,3}$ \\ 1 Department of Internal Medicine, National Taiwan University Hospital, Taipei 100, Taiwan; \\ chenpaoyu@gmail.com (P.-Y.C.); weischuang@gmail.com (Y.-C.C.); uninwu@gmail.com (U.-I.W.); \\ hysun@ntu.edu.tw (H.-Y.S.); wang.jt1968@gmail.com (J.-T.W.); whsheng@ntu.edu.tw (W.-H.S.); \\ changsc@ntu.edu.tw (S.-C.C.) \\ 2 Graduate Institute of Clinical Medicine, National Taiwan University College of Medicine, Taipei 100, Taiwan \\ 3 Department of Medicine, College of Medicine, National Taiwan University, Taipei 100, Taiwan \\ 4 National Health Research Institutes, Miaoli 350, Taiwan \\ * Correspondence: yeechunchen@gmail.com; Tel.: +886-2-23123456 (ext. 65054); Fax: +886-2-23971412
}

Citation: Chen, P.-Y.; Chuang, Y.-C.; Wu, U.-I.; Sun, H.-Y.; Wang, J.-T.; Sheng, W.-H.; Chen, Y.-C.; Chang, S.-C. Mechanisms of Azole Resistance and Trailing in Candida tropicalis Bloodstream Isolates. J. Fungi 2021, 7, 612. https://doi.org/10.3390/ jof7080612

Academic Editor: Ronen Ben-Ami

Received: 20 June 2021

Accepted: 26 July 2021

Published: 28 July 2021

Publisher's Note: MDPI stays neutral with regard to jurisdictional claims in published maps and institutional affiliations.

Copyright: () 2021 by the authors. Licensee MDPI, Basel, Switzerland. This article is an open access article distributed under the terms and conditions of the Creative Commons Attribution (CC BY) license (https:// creativecommons.org/licenses/by/ $4.0 /)$.

\begin{abstract}
Objectives: Azole-resistant Candida tropicalis has emerged in Asia in the context of its trailing nature, defined by residual growth above minimum inhibitory concentrations (MICs). However, limited investigations in C. tropicalis have focused on the difference of genotypes and molecular mechanisms between these two traits. Methods: Sixty-four non-duplicated C. tropicalis bloodstream isolates collected in 2017 were evaluated for azole MICs by the EUCAST E.def 7.3.1 method, diploid sequence type (DST) by multilocus sequencing typing, and sequences and expression levels of genes encoding ERG11, its transcription factor, UPC2, and efflux pumps (CDR1, CDR2 and MDR1). Results: Isavuconazole showed the highest in vitro activity and trailing against $C$. tropicalis, followed by voriconazole and fluconazole (geometric mean [GM] MIC, 0.008, 0.090, $1.163 \mathrm{mg} / \mathrm{L}$, respectively; trailing GM, $27.4 \%, 20.8 \%$ and $19.5 \%$, respectively; both overall $p<0.001)$. Fourteen $(21.9 \%)$ isolates were non-WT to fluconazole/voriconazole, 12 of which were non-WT to isavuconazole and clustered in clonal complex (CC) 3. Twenty-five (39.1\%) isolates were high trailing WT, including all CC2 isolates $(44.0 \%$ ) (containing DST140 and DST98). All azole non-WT isolates carried the ERG11 mutations A395T/W and/or C461T/Y, and most carried the UPC2 mutation T503C/Y. These mutations were not identified in low and high trailing WT isolates. Azole non-WT and high trailing WT isolates exhibited the highest expression levels of ERG11 and MDR1, 3.91- and 2.30-fold, respectively (both overall $p<0.01)$. Conclusions: Azole resistance and trailing are phenotypically and genotypically different in C. tropicalis. Interference with azole binding and MDR1 up-regulation confer azole resistance and trailing, respectively.
\end{abstract}

Keywords: fluconazole; voriconazole; isavuconazole; multilocus sequence typing; ergosterol; efflux pump

\section{Introduction}

Candida tropicalis is among the four major Candida species responsible for candidaemia worldwide [1]. It is the most common aetiology of invasive candidiasis in patients with haematological malignancies. The proportion of C. tropicalis among Candida species causing candidaemia are relatively high in tropical Asia and Latin America compared to other continents [2,3]. Azole-resistant C. tropicalis clinical isolates have emerged worldwide [4-11]. This has become particularly problematic in the Asia-Pacific region since 2010 [4,5,7,10,11]. Clonal complex 3 (CC3) with high-level azole resistance were isolated from patients and environments $[5,7,10,11]$.

Explorations into molecular mechanisms of azole resistance in C. tropicalis include mutations and/or up-regulation of ERG11 gene encoding the azole target lanosterol 14- $\alpha$ - 
sterol-demethylase [6,9,12-15], gain-of-function mutations in UPC2 gene which encodes a transcription factor of ERG11 [8,16], and overexpression of genes encoding efflux pumps, including ATP binding cassette (ABC) transporters and major facilitator superfamily (MFS), including MDR1 [13-16]. C. tropicalis has two ABC transporters (CDR1 and CDR2) [17], while the role of $C D R 2$ in azole resistance is less characterized. These resistance mechanisms were also found in other Candida species, including C. albicans and C. glabrata $[18,19]$.

Azole trailing is defined as reduced, but with persistent growth at azole concentrations above the minimum inhibitory concentrations (MICs) [20-22]. Specifically, Candida species display growth inhibition of $50-80 \%$ over a broad azole concentration range, defined as trailing growth [20-22], according to current European Committee on Antimicrobial Susceptibility Testing (EUCAST) and Clinical and Laboratory Standard Institute (CLSI) recommending that the MIC of azole against Candida species is determined after $24 \mathrm{~h}$ at the lowest drug concentration giving growth inhibition of $\geq 50 \%$ of that of the drug-free control. It is often difficult to distinguish between azole trailing and resistance, therefore, the European Committee on Antimicrobial Susceptibility Testing (EUCAST) broth microdilution (BMD) method recommended the spectrophotometric endpoint reading to minimize the impact of azole trailing on MIC determinations [23].

In addition to C. albicans and C. glabrata, C. tropicalis is one of Candida species commonly displaying azole trailing $[20,21,24]$, while the degree of trailing among different azoles is seldom compared in C. tropicalis. Although azole trailing isolates appear to be azole susceptible by in vitro testing, the molecular mechanisms involving azole resistance have been observed in C. albicans trailing isolates [25]. However, evidence of whether the aforementioned resistance mechanisms also play roles in trailing is limited [22]. Clonality of $C$. tropicalis with azole trailing was less investigated and the results in the previous studies were contradictory $[9,20]$.

This study was designed to determine the activity of different azoles and their degree of trailing for C. tropicalis bloodstream isolates using the EUCAST BMD method to ascertain their clonal relationships, and to compare the molecular mechanisms between azole resistance and trailing isolates.

\section{Materials and Methods}

\subsection{Candida Tropicalis Isolates}

We evaluated 64 non-duplicated C. tropicalis bloodstream isolates from patients with candidaemia admitted to the National Taiwan University Hospital in 2017 [7]. These isolates were identified by matrix-assisted laser desorption ionization-time of flight mass spectrometry (Bruker, Bremen, Germany) in the central laboratory. They were reconfirmed for this study using CHROMagar Candida (Becton, Dickinson and Company, Sparks, MD, USA), the Vitek 2 yeast identification systems (bioMérieux, Marcy-L'Etoile, France), and/or internal transcribed spacer (ITS) sequencing as previously described [7]. This study was approved by the Research Ethics Committees (NTUH-201502034RIND, 201805097RIND).

\subsection{Antifungal Susceptibility Testing and Trailing}

The MICs for fluconazole, voriconazole (Sigma, St. Louis, MO, USA) and isavuconazole (Toronto Research Chemicals, Toronto, ON, Canada) were determined according to the EUCAST E.Def 7.3.1 method [20] using Costar tissue culture-treated plates (catalog no. CLS3596; Corning Life Sciences, Wujiang, China). Ten dilution ranges of each azole were prepared according to ISO recommendations. Candida parapsilosis (ATCC 22019) and Candida krusei (ATCC 6258) were used as reference strains for quality control. The MICs of fluconazole and voriconazole for $C$. tropicalis were interpreted according to the EUCAST epidemiological cut-off values (ECOFFs) for wild-type (WT)/non-WT classification, $1 \mathrm{mg} / \mathrm{L}$ and $0.125 \mathrm{mg} / \mathrm{L}$, respectively. The tentative ECOFF of isavuconazole in this report is $0.03 \mathrm{mg} / \mathrm{L}$, which was categorized based on isavuconazole MICs > two dilutions above the $\mathrm{MIC}_{50}$ [26]. Azole trailing was calculated as the geometric mean of residual growth observed in the wells in which concentrations were above the MICs compared to 
the growth control (background OD subtracted) [21]. WT isolates were dichotomized as low and high trailing groups by the median percentage of trailing among them: $19.9 \%$, $21.5 \%$, and $28.5 \%$ for fluconazole, voriconazole, and isavuconazole, respectively.

\subsection{Multilocus Sequence Typing}

C. tropicalis genomic DNA was extracted and all isolates were typed using an MLST scheme described previously [7]. In brief, the sequences of the nucleotide primers were used for PCR amplification of six gene fragments. Combination of the results from the six gene fragments yielding unique DSTs were used to infer putative genetic relationships between $C$. tropicalis isolates using the goeBURST algorithm version 1.2.1 [27].

\subsection{ERG11 and UPC2 Sequencing}

C. tropicalis genomic DNA was used as the template for the amplification of the fulllength coding region of the ERG11 and UPC2 genes. The primers for amplification and sequencing were designed as previously described with modifications (Supplementary Table S1) [28-30]. Nucleotide sequences of the amplicon were aligned with those of the ERG11 gene of C. tropicalis ATCC 750 (GenBank accession number: M23673.1) and with those of the UPC2 gene of $C$. tropicalis MYA-3404 (GenBank accession number: NW_003020056.1).

\subsection{Quantitative RT-PCR}

Total RNA was extracted from each isolate grown to the mid exponential phase $\left(1 \times 10^{7}\right.$ cells $\left./ \mathrm{mL}\right)$ in YPD medium using the Qiagen 74104 RNeasy Mini Kit (Qiagen Inc., Germantown, MD, USA) following the manufacturer's instructions. Total RNA was quantified using Nanodrop 2000 (Thermo Fisher Scientific, Waltham, MA, USA). First-strand cDNA was synthesized from 50 ng of total RNA using a GoScript ${ }^{\text {TM }}$ Reverse Transcription System (Promega A5000, Madison, WI, USA). Quantitative PCR of ACT1, ERG11, UPC2, CDR1, CDR2, and MDR1 was performed in triplicate using the Power SYBR $^{\circledR}$ green PCR master (Thermo Fisher Scientific, Waltham, MA, USA) and an Applied Biosystems StepOnePlus (Applied Biosystems, Foster City, CA, USA). The primers used for quantitative RT-PCR were listed in Supplementary Table S1. Amplification conditions consist of $10 \mathrm{~min}$ of denaturation at $95^{\circ} \mathrm{C}$, followed by 40 cycles of $15 \mathrm{~s}$ at $95{ }^{\circ} \mathrm{C}$ and $60 \mathrm{~s}$ at $60^{\circ} \mathrm{C}$. The expression levels were normalized by $A C T 1$ expression. Relative gene expressions were calculated as the fold change of each isolate compared with the median expression levels of low trailing WT isolates.

\subsection{Statistical Analysis}

Geometric means of azole MICs, trailing and proportions of prior azole exposure within 30 days before onset of candidaemia were compared among low trailing WT, high trailing WT, and non-WT isolates using the one-way ANOVA with Bonferroni correction, while relative gene expressions among the three groups were compared with KruskalWallis with Dunn's multiple comparisons test. Correlations between azole MICs were calculated using Pearson correlation analyses after $\log 2$ transformation, and those between azole trailing and MDR1 expressions were calculated using the Spearman correlation analysis. All analyses were performed using Stata software (version 14; StataCorp, College Station, TX, USA). Two-sided $p$ values less than 0.05 were considered significant.

\section{Results}

\subsection{Azole Minimum Inhibitory Concentrations, Trailing, and Corresponding Genotypes}

Among 64 C. tropicalis bloodstream isolates, isavuconazole was most active followed by voriconazole, and then fluconazole regarding $\mathrm{MIC}_{90}$ and geometric means of MICs (Table 1). Of 14 isolates (21.9\%) classified as non-wild-type (WT) to fluconazole and voriconazole, 12 isolates were also classified as non-WT to isavuconazole. All azole nonWT isolates were categorized as resistant to fluconazole/voriconazole by EUCAST clinical breakpoints. The strongest correlation was observed between fluconazole MIC and 
voriconazole MIC ( $R^{2}$ of 0.967$)$, followed by those between isavuconazole and voriconazole/fluconazole $\left(R^{2}\right.$ of 0.766 and 0.719 , respectively; All $\left.p<0.001\right)$.

Table 1. Minimum inhibitory concentrations (mg/L), proportions of non-wild-type, and percentage of trailing for different azoles against 64 Candida tropicalis bloodstream isolates.

\begin{tabular}{|c|c|c|c|c|c|c|c|}
\hline & \multirow{2}{*}{$\begin{array}{l}\text { Fluconazole } \\
\text { (FLC) }\end{array}$} & \multirow{2}{*}{$\begin{array}{l}\text { Voriconazole } \\
\text { (VRC) }\end{array}$} & \multirow{2}{*}{$\begin{array}{l}\text { Isavuconazole } \\
\text { (ISA) }\end{array}$} & \multicolumn{4}{|c|}{$p$-Value } \\
\hline & & & & Overall & $\begin{array}{l}\text { FLC vs. } \\
\text { VRC }\end{array}$ & $\begin{array}{l}\text { FLC vs. } \\
\text { ISA }\end{array}$ & $\begin{array}{l}\text { VRC vs. } \\
\text { ISA }\end{array}$ \\
\hline MIC Range a,b & $\leq 0.125->64$ & $\leq 0.015-4$ & $\leq 0.002-0.125$ & & & & \\
\hline $\mathrm{MIC}_{50} / \mathrm{MIC}_{90}$ & $0.5 />64$ & $0.06 / 4$ & $0.008 / 0.125$ & & & & \\
\hline GM MIC & 1.163 & 0.090 & 0.008 & $<0.001$ & $<0.001$ & $<0.001$ & $<0.001$ \\
\hline$\%$ of Non-WT & & & & & & & \\
\hline $\begin{array}{l}{ }^{\mathrm{c}} \text { Isolates(No. } \\
\text { of Isolates) }\end{array}$ & $21.9(14)$ & $21.9(14)$ & $18.8(12)$ & & & & \\
\hline $\begin{array}{c}\text { GM }( \pm S D) \text { of } \\
\text { Trailing }(\%)\end{array}$ & $19.5( \pm 7.9)^{d}$ & $20.8( \pm 6.4)$ & $27.4( \pm 6.3)$ & $<0.001$ & $>0.99$ & $<0.001$ & $<0.001$ \\
\hline
\end{tabular}

Abbreviations: $\mathrm{MIC}_{50} / \mathrm{MIC}_{90}$, MIC for $50 \%$ and $90 \%$ of the respective population; GM, geometric mean; non-WT, non-wild-type; SD, standard deviation. ${ }^{a}$ The 10-dilution range of each azole are listed as follows: fluconazole, $\leq 0.125 \mathrm{mg} / \mathrm{L}-64 \mathrm{mg} / \mathrm{L}$; voriconazole, $\leq 0.015$ $\mathrm{mg} / \mathrm{L}-8 \mathrm{mg} / \mathrm{L}$; isavuconazole, $\leq 0.002 \mathrm{mg} / \mathrm{L}-1 \mathrm{mg} / \mathrm{L} .{ }^{\mathrm{b}}$ Quality control strains were tested two times, and results for each azole against each quality control strain are as follows: C. krusei ATCC 6258, fluconazole, $32 \mathrm{mg} / \mathrm{L}$ (twice) (target [range] 32 [16-64] mg/L); voriconazole $0.25 \mathrm{mg} / \mathrm{L}$ (twice) $(0.06-0.125$ [0.03-0.25] mg/L); isavuconazole, 0.03 and $0.06 \mathrm{mg} / \mathrm{L}$ (once for each) $(0.03$ [0.016-0.06] mg/L); C. parapsilosis ATCC 22019, fluconazole, 0.5 and $1 \mathrm{mg} / \mathrm{L}$ (once for each) $(1$ [0.5-2] mg/L); voriconazole $0.03 \mathrm{mg} / \mathrm{L}$ (twice) $(0.03$ [0.016-0.06] mg/L); isavuconazole, $0.008 \mathrm{mg} / \mathrm{L}$ (twice) $\left(0.016\right.$ [0.008-0.03] mg/L). ${ }^{c}$ The current EUCAST epidemiological cut-off values (ECOFFs) of fluconazole and voriconazole for C. tropicalis are $1 \mathrm{mg} / \mathrm{L}$ and $0.125 \mathrm{mg} / \mathrm{L}$. The tentative ECOFF of isavuconazole in this report is $0.03 \mathrm{mg} / \mathrm{L}$. ${ }^{\mathrm{d}}$ Isolates with fluconazole MICs $\geq 64 \mathrm{~m} / \mathrm{L}$ were excluded from the calculating percentage of trailing due to out-of-dilution ranges.

Overall, isavuconazole displayed the highest mean percentages of trailing, followed by voriconazole and fluconazole (Table 1), and the orders of trailing degree among azoles remained similar in low and high trailing WT and non-WT subgroups (Figure 1). Additionally, the degree of trailing for each azole was highest in high trailing WT isolates compared to those in non-WT and low trailing WT isolates (Figure 1), suggesting high trailing was not misclassified as non-WT.

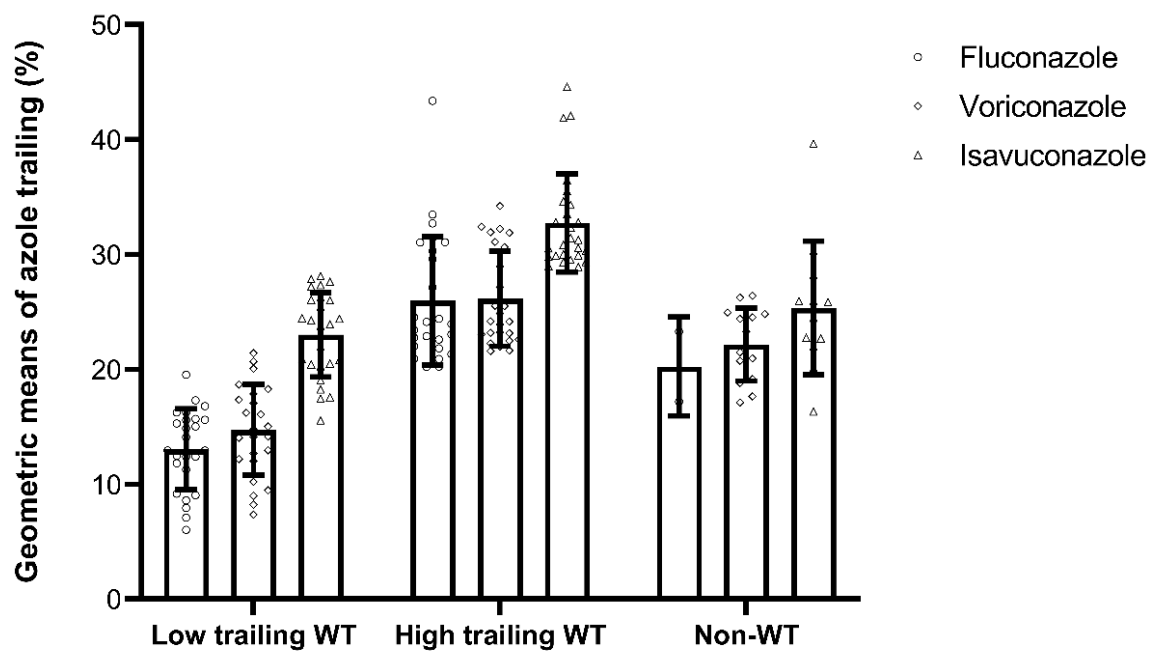

Figure 1. Geometric means of trailing percentages for fluconazole, voriconazole, and isavuconazole among low and high trailing WT and non-WT C. tropicalis isolates. Isolates with fluconazole MICs of $\geq 64 \mathrm{~m} / \mathrm{L}$ were excluded from calculating the percentage of trailing due to out-of-dilution ranges. Error bars indicate standard deviations.

As shown in Table 2 and Supplementary Figure S1, azole non-WT isolates were mainly within CC3, none of which displayed trailing growth (Supplementary Figure S2). This suggested fluconazole non-WT in this clone was not attributed to heavy trailing growth 
of $>50 \%$, also called resistant heavily trailing, over a broad fluconazole concentration range [22]. On the other hand, all $11 \mathrm{CC} 2$ isolates were classified as high trailing WT to three azoles, except one as a low trailing WT to isavuconazole, and contributed $44.0 \%$ of high trailing to fluconazole/voriconazole WT isolates. Of note, fluconazole/voriconazole non-WT isolates had a greatest proportion of prior azole exposure within 30 days before candidaemia onset, followed by those in high and low trailing WT isolates (57.1\% vs. $28.0 \%$ vs. $4.0 \%$, overall $p<0.001$ ).

Table 2. Distributions of low and high trailing wild-type, and non-wild-type Candida tropicalis isolates, and their corresponding clonal complexes ${ }^{a}$ and minimum inhibitory concentrations $(\mathrm{mg} / \mathrm{L})$.

\begin{tabular}{|c|c|c|c|c|c|c|c|c|c|}
\hline & \multicolumn{3}{|c|}{ Fluconazole } & \multicolumn{3}{|c|}{ Voriconazole } & \multicolumn{3}{|c|}{ Isavuconazole } \\
\hline & $\begin{array}{c}\text { Low } \\
\text { Trailing } \\
\text { WT }\end{array}$ & $\begin{array}{c}\text { High } \\
\text { Trailing } \\
\text { WT }\end{array}$ & $\begin{array}{l}\text { Non- } \\
\text { WT }\end{array}$ & $\begin{array}{c}\text { Low } \\
\text { Trailing } \\
\text { WT }\end{array}$ & $\begin{array}{c}\text { High } \\
\text { Trailing } \\
\text { WT }\end{array}$ & $\begin{array}{l}\text { Non- } \\
\text { WT }\end{array}$ & $\begin{array}{c}\text { Low } \\
\text { Trailing } \\
\text { WT }\end{array}$ & $\begin{array}{c}\text { High } \\
\text { Trailing } \\
\text { WT }\end{array}$ & $\begin{array}{l}\text { Non- } \\
\text { WT }\end{array}$ \\
\hline \multicolumn{10}{|l|}{$\begin{array}{c}\text { Clonal } \\
\text { complex }{ }^{b} \text { (No. } \\
\text { of Isolates) }\end{array}$} \\
\hline $1(4)$ & 4 & 0 & 0 & 4 & 0 & 0 & 4 & 0 & 0 \\
\hline $2(11)$ & 0 & 11 & 0 & 0 & 11 & 0 & 1 & 10 & 0 \\
\hline $3(14)$ & 2 & 0 & 12 & 0 & 2 & 12 & 0 & 2 & 12 \\
\hline $4(10)$ & 5 & 5 & 0 & 6 & 4 & 0 & 4 & 6 & 0 \\
\hline $6(2)$ & 1 & 1 & 0 & 2 & 0 & 0 & 1 & 1 & 0 \\
\hline $8(2)$ & 0 & 2 & 0 & 0 & 2 & 0 & 0 & 2 & 0 \\
\hline $9(2)$ & 2 & 0 & 0 & 2 & 0 & 0 & 2 & 0 & 0 \\
\hline $11(1)$ & 0 & 0 & 1 & 0 & 0 & 1 & 1 & 0 & 0 \\
\hline $20(2)$ & 2 & 0 & 0 & 1 & 1 & 0 & 2 & 0 & 0 \\
\hline $22(3)$ & 2 & 1 & 0 & 2 & 1 & 0 & 1 & 2 & 0 \\
\hline $28(1)$ & 1 & 0 & 0 & 1 & 0 & 0 & 1 & 0 & 0 \\
\hline $35(1)$ & 1 & 0 & 0 & 1 & 0 & 0 & 1 & 0 & 0 \\
\hline $41(1)$ & 0 & 0 & 1 & 0 & 0 & 1 & 0 & 1 & 0 \\
\hline $49(1)$ & 1 & 0 & 0 & 1 & 0 & 0 & 1 & 0 & 0 \\
\hline $63(1)$ & 0 & 1 & 0 & 0 & 1 & 0 & 1 & 0 & 0 \\
\hline Singletons (8) & 4 & 4 & 0 & 5 & 3 & 0 & 6 & 2 & 0 \\
\hline Total (64) & 25 & 25 & 14 & 25 & 25 & 14 & 26 & 26 & 12 \\
\hline \multicolumn{10}{|l|}{$\mathrm{MIC}^{\mathrm{c}}$} \\
\hline GM & 0.330 & 0.379 & $>64$ & 0.269 & 0.418 & 3.123 & 0.004 & 0.006 & 0.104 \\
\hline $\mathrm{MIC}_{50}$ & 0.25 & 0.5 & $>64$ & 0.03 & 0.06 & 4 & 0.006 & 0.008 & 0.125 \\
\hline $\mathrm{MIC}_{90}$ & 0.5 & 0.5 & $>64$ & 0.06 & 0.06 & 4 & 0.015 & 0.015 & 0.125 \\
\hline Range & $\begin{array}{c}\leq 0.125- \\
0.5\end{array}$ & $\begin{array}{c}\leq 0.125- \\
0.5\end{array}$ & $\begin{array}{c}8- \\
>64\end{array}$ & $\begin{array}{c}\leq 0.015- \\
0.06\end{array}$ & $\begin{array}{c}\leq 0.015- \\
0.06\end{array}$ & $0.5-4$ & $\begin{array}{c}\leq 0.002- \\
0.015\end{array}$ & $\begin{array}{c}\leq 0.002- \\
0.015\end{array}$ & $\begin{array}{l}0.06- \\
0.125\end{array}$ \\
\hline
\end{tabular}

Abbreviations: DST, diploid sequence type; non-WT, non-wild-type; MIC, minimum inhibitory concentration; GM, geometric men; $\mathrm{MIC}_{50} / \mathrm{MIC}_{90}$, MIC for $50 \%$ and $90 \%$ of the; MIC, minimum inhibitory concentration; GM, geometric men; $\mathrm{MIC}_{50} / \mathrm{MIC}_{90}$, MIC for $50 \%$ and $90 \%$ of the respective population. ${ }^{\text {a }}$ The most prevalent wild-type clone with high trailing is highlighted in boldface type, and the most dominant non-wild-type (WT) clone is highlighted with gray shading. ${ }^{b}$ Clonal complex is determined by multilocus sequence typing when five of the six alleles are identical between a pair as described previously [7]. In this study, CC2 included diploid sequence type (DST) 98 $(n=3)$, DST140 $(n=5)$, DST820 $(n=1)$, DTS829 $(n=1)$, and DST830 $(n=1)$. Twelve of 14 isolates in CC3 classified as non-WT to three tested azoles included DST376, DST505, DST507, and DST838. The other two in CC3 categorized as WT to three tested azoles included DST333 and DST847 as described previously [7]. ' Quality control strains were tested 2 times, and results for each azole against each quality control strain were as followed: C. krusei ATCC 6258, fluconazole, $32 \mathrm{mg} / \mathrm{L}$ (twice) (target [range] 32 [16-64] mg/L); voriconazole $0.25 \mathrm{mg} / \mathrm{L}$ (twice) (0.06-0.125 [0.03-0.25] mg/L); isavuconazole, 0.03 and $0.06 \mathrm{mg} / \mathrm{L}$ (once for each) $(0.03$ [0.016-0.06] mg/L); C. parapsilosis ATCC 22019 , fluconazole, 0.5 and $1 \mathrm{mg} / \mathrm{L}$ (once for each) $(1[0.5-2] \mathrm{mg} / \mathrm{L}$ ); voriconazole $0.03 \mathrm{mg} / \mathrm{L}$ (twice) $(0.03$ [0.016-0.06] mg/L); isavuconazole, 0.008 (twice) $(0.016[0.008-0.03] \mathrm{mg} / \mathrm{L})$.

\subsection{ERG11 and UPC2 Sequencing}

Twelve azole non-WT CC3 isolates possessed homozygote and/or heterozygote nonsynonymous mutations, A395T/W and C461T/Y (Table 3), corresponding to Y132F and S154F substitutions, respectively. Only one nonsynonymous mutation in the UPC2 gene, T503Y/C, was exclusively detected in azole non-WT CC3 isolates. These isolates displayed 
high fluconazole and voriconazole MICs of $\geq 64 \mathrm{mg} / \mathrm{L}$ and of $4 \mathrm{mg} / \mathrm{L}$, respectively, and non-WT to isavuconazole. The remaining two azole non-WT non-CC3 isolates sharing A395T/W and/or C461T/Y displayed relatively low fluconazole and voriconazole MICs (8-16 mg/L, and 0.5-1 mg/L, respectively), and WT to isavuconazole. All low and high trailing WT isolates did not have the above ERG11 and UPC2 mutations.

Table 3. Homozygote and heterozygote nonsynonymous mutations in ERG11 gene and UPC2 gene among 14 ERG11 non-wild-type Candida tropicalis isolates with azole MICs and corresponding clonal complexes.

\begin{tabular}{|c|c|c|c|c|c|c|c|}
\hline \multirow[b]{2}{*}{$\begin{array}{l}\text { Nucleotide } \\
\text { Position }\end{array}$} & \multicolumn{2}{|c|}{ ERG11 Gene ${ }^{a}$} & \multirow{2}{*}{$\begin{array}{c}\text { UPC2 Gene } \\
503\end{array}$} & \multirow{2}{*}{$\begin{array}{l}\text { Clonal Complex } \\
\text { (No. of Isolates) }\end{array}$} & \multicolumn{3}{|c|}{ MIC Range $(\mathrm{mg} / \mathrm{L})^{d}$} \\
\hline & 395 & 461 & & & FLC & VRC & ISA \\
\hline & $\mathrm{A}$ & $\mathrm{C}$ & $\mathbf{T}$ & Reference $^{b}$ & & & \\
\hline & $\mathbf{W}$ & $\mathbf{Y}$ & $\mathbf{Y}$ & $\mathrm{CC} 3^{\mathrm{c}}(11)$ & $>64$ & 4 & $0.06-0.125$ \\
\hline & $\mathbf{W}$ & $\mathbf{Y}$ & $\mathrm{C}$ & $\mathrm{CC} 3^{\mathrm{c}}(1)$ & 64 & 4 & 0.125 \\
\hline & $\mathrm{T}$ & $\mathbf{T}$ & $\mathrm{T}$ & $\mathrm{CC} 41^{\mathrm{c}}(1)$ & 16 & 1 & 0.015 \\
\hline & $\mathrm{A}$ & $\mathbf{T}$ & $\mathrm{T}$ & $\mathrm{CC} 11^{\mathrm{c}}(1)$ & 8 & 0.5 & 0.008 \\
\hline
\end{tabular}

Abbreviations: MIC, minimum inhibitory concentrations; FLC, fluconazole; VRC, voriconazole; ISA, isavuconazole; CC, clonal complex. a Nonsynonymous mutations are highlighted in boldface. Heterozygotes $(K, M, R, S, W$, and $Y$ ) are defined by the International Union of Pure and Applied Chemistry (https:/ /iupac.org; accessed on 30 August 2020) nomenclature. The meaning of R, W, and Y refer to AG, $\mathrm{AT}$, and CT, respectively. ${ }^{\mathrm{b}}$ Reference strain sequence: ERG11 gene: ATCC750, GeneBank accession no.: M23673.1; UPC2 gene: MYA3404, GenBank accession no.: NW_003020056.1. ${ }^{c}$ Clonal complex is determined by multilocus sequence typing when five of the six alleles are identical between a pair as described previously [7]. In this study, 12 isolates in CC3 were classified as non-wild-type (WT) to three tested azoles, including the diploid sequence type (DST) 376, DST505, DST507, and DST838. Two isolates classified as non-WT to fluconazole and voriconazole, but WT to isavuconazole, belonged to DST508 in CC11, and DST819 in CC41, respectively. ${ }^{\mathrm{d}}$ Quality control strains were tested 2 times, and results for each azole against each quality control strain were as followed: C. krusei ATCC 6258, fluconazole, $32 \mathrm{mg} / \mathrm{L}$ (twice) (target [range] 32 [16-64] mg/L); voriconazole $0.25 \mathrm{mg} / \mathrm{L}$ (twice) $(0.06-0.125$ [0.03-0.25] mg/L); isavuconazole, 0.03 and $0.06 \mathrm{mg} / \mathrm{L}$ (once for each) (0.03 [0.016-0.06] mg/L); C. parapsilosis ATCC 22019, fluconazole, 0.5 and $1 \mathrm{mg} / \mathrm{L}$ (once for each) (1 [0.5-2] $\mathrm{mg} / \mathrm{L}$ ); voriconazole $0.03 \mathrm{mg} / \mathrm{L}$ (twice) $(0.03$ [0.016-0.06] mg/L); isavuconazole, 0.008 (twice) $(0.016$ [0.008-0.03] mg/L).

\subsection{Expression of ERG11, UPC2, and Genes Encoding Efflux Pumps}

Gene expression levels of ERG11 and UPC2 differed significantly among azole non-WT isolates and low and high trailing WT isolates (both overall $p<0.05$ ) (Figure 2A). Azole non-WT isolates exhibited 3.91-fold higher median expression levels of the ERG11 gene than those of low and high trailing WT isolates (both $p<0.001$ ) (Figure 2A).

Azole non-WT isolates exhibited the highest and lowest median expression levels of the CDR2 and CDR1 genes compared to low and high trailing WT isolates, while there was no difference between those in low and high trailing WT isolates (Figure 2B). On the other hand, high trailing WT isolates exhibited the highest median expression levels (2.30-fold) of MDR1 than those of low trailing WT and non-WT isolates (overall and pair-wised $p<0.05)$. Furthermore, the degree of trailing was positively correlated with MDR1 expression levels ( $\mathrm{r}=0.346 ; p=0.005)$ (Supplementary Figure S3).

Among 14 azole non-WT isolates, CC3 isolates demonstrated higher expression levels of ERG11 but similar expression levels of other genes compared to those of non-CC3 isolates (Figure 2A,B). 
(A)

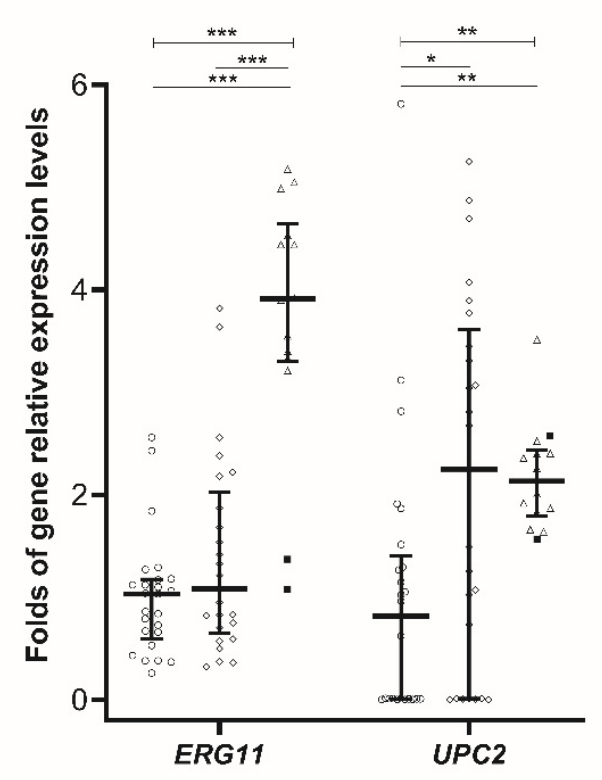

Low trailing WT

- High trailing WT

$\triangle$ Non-WT CC3

- Non-WT non-CC3

(B)

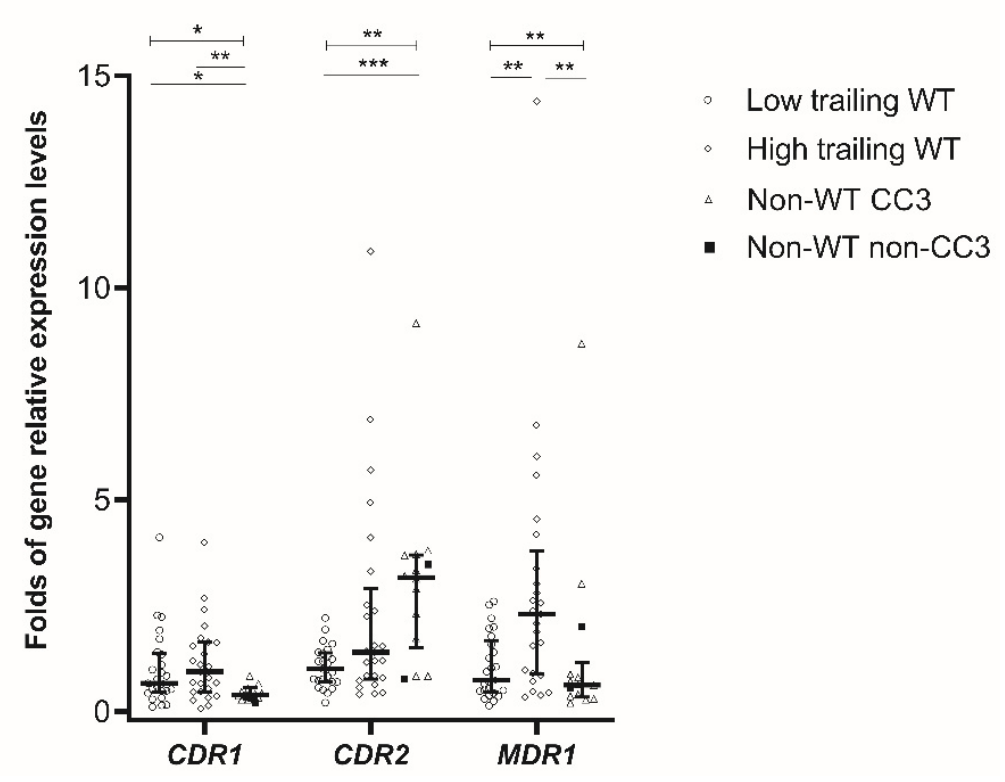

Figure 2. Expression levels of genes encoding the azole target or its transcription factor, ERG11 and UPC2 (A), and genes encoding efflux pumps, CDR1, CDR2, and MDR1 (B) of low and high trailing WT, and non-WT C. tropicalis isolates. Horizontal lines and error bars indicate medians and interquartile, respectively. Asterisks indicate statistical significance $\left(^{*}: p<0.05 ;{ }^{* *}: p<0.01 ; * * *: p<0.001\right)$.

\section{Discussion}

This study confirmed previous studies that isavuconazole was most potent against C. tropicalis among three azoles evaluated while its non-WT showed cross-resistance to fluconazole and voriconazole [26,31]. Isavuconazole displayed the highest degree of trailing among different azoles against $C$. tropicalis $[20,21]$. This study distinguished azole nonWT from high trailing WT and demonstrated that genotypes and molecular mechanisms varied between them. Azole non-WT isolates had nonsynonymous mutations and/or overexpression of the ERG11 gene, alongside with up-regulations of $C D R 2$, while upregulation of MDR1 was mainly found in high trailing WT isolates.

Overall, in vitro activities for three azoles tested against $C$. tropicalis in this study were consistent with previously published EUCAST MICs [21,26,31]. Given isavuconazole shares 
more similar chemical structures with short-chained azoles, our and other reports [26] demonstrated good correlation between isavuconazole and fluconazole/voriconazole. This is of particular concern in view of the emerging pan-azole-resistant clone, CC3, in Asia $[5,7,10,11]$. As shown in this study, $C$. tropicalis isolates exhibiting cross-resistance between isavuconazole and other azoles were collected in 2017, while isavuconazole was introduced in Asia in 2019. As antifungal susceptibility testing is not routinely performed in many Asian hospitals [32] and azoles are step-down oral agents for invasive candidiasis, clinicians should be aware of the possibility of pan-azole resistance in C. tropicalis.

Azole trailing in Candida is a common phenomenon which has been evaluated by various methodologies and reading endpoints (24 vs. 48 h) $[9,20-22,24,25,33,34]$. Our results were consistent with previous reports that quantification of trailing among different Candida strains or antifungal agents was feasible by performing the $24 \mathrm{~h}$ EUCAST BMD method [20-22]. Marcos-Zambrano L.J. et al. showed C. tropicalis displayed a higher degree of trailing to fluconazole and isavuconazole among different Candida species [20,21]. We further demonstrated that $C$. tropicalis produced the highest degree of trailing to isavuconazole among various azoles, and those to fluconazole and voriconazole were comparable.

CC2 was most prevalent among high trailing WT isolates in a diverse genetic background (Table 2 and Supplementary Figure S1). DST140 fluconazole WT isolate was first identified in 1987 at this hospital (data not shown). Later, another Taiwan surveillance study in 1999 demonstrated fluconazole trailing growth clustered in the CC2 clone, mainly containing DST98 and DST140 [34]. Additionally, our previous study has demonstrated CC2 as the second largest clone after CC 3 without expansion by nosocomial transmission from 2011 to 2017 in Taiwan [7]. Whether this trailing nature is genotype-specific warrants further exploration, but our findings and others $[9,20,34]$ suggest that geographic variation in C. tropicalis genotypes should be considered for variations in trailing growth from different studies.

As for molecular mechanisms of azole resistance, this study found that all 14 azole non-WT isolates had the specific ERG11 mutations, which supports the current EUCAST fluconazole/voriconazole ECOFFs for C. tropicalis. These ERG11 mutations resulting in Y132F and/or S154F substitutions were concordant to previous studies [9,12-15]. Y132F alternation is located on the exposed active-site cavity of the Erg11 protein. This results in interference with azole binding and subsequent azole resistance across different Candida spp., including multi-drug resistant Candida auris and C. albicans [17-19,35]. A Saccharomyces cerevisiae model also has confirmed that $\mathrm{Y} 132 \mathrm{~F}$ can cause azole resistance in C. tropicalis [12,14].

In this report, wide variations of fluconazole MICs among azole non-WT isolates may indicate that ERG11 mutations per se do not fully account for azole resistance levels. In this study, coexistence of ERG11 overexpression and ERG11 mutations in CC3 conferred high-level resistance to fluconazole/voriconazole and non-WT to isavuconazole, while the other two azole non-WT non-CC3 isolates in the absence of ERG11 overexpression showed moderate resistance to fluconazole/voriconazole and WT to isavuconazole. Given we found only CC3 isolates carried the UPC2 mutation T503C/Y and ERG11 overexpression and other studies also showed specific UPC2 mutations in azole-resistant $C$. tropicalis $[8,16]$, we speculated the gain-of-function mutations of UPC2 may play roles in regulating ERG11 expression.

Overexpression of different efflux pumps, either CDR1 or MDR1, in azole-resistant $C$. tropicalis has been reported previously $[13,15,16,22]$, while our study was first to demonstrate up-regulations of CDR2 and MDR1 expression in azole non-WT and high trailing WT isolates, respectively. This is concordant to a S. cerevisiae model showing ABC transporters, but not MDR1 conferring azole resistance in C. albicans and C. glabrata [35]. Additionally, a previous study demonstrated up-regulation of $A B C$ transporters not related to the degree of trailing in C. tropicalis [22]. Our finding, showing the positive correlation between the degree of trailing and MDR1 expression levels in C. tropicalis, also supported the role of MFS up-regulation in azole trailing.

This finding further suggests trailing is a unique trait depending on azole induction. This in conjunction with the Galleria model showing reduced fluconazole activities against 
both moderate and heavy azole trailing C. tropicalis isolates (defined by trailing of $26-50 \%$ and $>50 \%$, respectively) [22] raised the concern about the efficacy of azole in treatment for azole trailing C. tropicalis infections. Although the Galleria model did not show a significant survival difference between voriconazole trailing and susceptible C. albicans strains as treated by voriconazole [36], clinical studies have shown azole tolerant $C$. albicans cause persistent candidaemia or higher mortalities $[33,37]$. However, clinical outcomes of candidaemia patients infected by azole trailing C. tropicalis were not different, even better, compared to those infected by azole non-trailing isolates in relatively small cohorts $[9,38]$. Hence, this is warranted to determine the impact of azole trailing strains on patients with C. tropicalis bloodstream infections to guide treatment choices.

Our study had some limitations. First, our observations were generated mainly based on a unique clonal complex of azole non-WT and high trailing WT. These findings are likely unable to be generalized to other countries in the absence of the characterization of genotypes of $C$. tropicalis. Second, heavily trailing $(>50 \%)$ C. tropicalis not identified in this study also limits generalization of our results. Also, the threshold value to define high and low trailing WT is specific for the collection of isolates used in this study, it may not be applicable to other laboratories. Additionally, our findings may not be generalized to C. albicans given other studies have shown both $A B C$ transports and MFS up-regulation confer azole resistance in C. albicans $[18,19,39]$. We examined the potential mechanisms of azole resistance and trailing in C. tropicalis as thoroughly as possible, but molecular mechanisms of these two distinct traits are more complicated. For example, genomic structure variations, either aneuploidy or copy number variations of drug resistant genes, also confer azole resistance and/or trailing $[17,40]$. Notably, mutations of transcription factors regulating efflux pumps, including TAC1 and MRR1, have been identified in azole resistance C. tropicalis [9], while their roles in azole trailing have yet to be defined. Furthermore, efflux pump activities in azole trailing may only reflect the downstream phenomenon of variable stress responses as Candida species expose to azoles [33].

Collectively, our results supported not only EUCAST fluconazole/voriconazole ECOFFs for C. tropicalis, but also the EUCAST BMD method to quantify the degree of azole trailing. Furthermore, our results underscored azole resistance and trailing of $C$. tropicalis are phenotypically and genotypically different as shown in C. albicans. Most azole non-WT isolates belong to CC 3 , while CC2 is the most prevalent clone among high trailing WT isolates. Interference with azole binding and MDR1 up-regulation were the major mode of resistance and the degree of trailing, respectively. Deep insights to underlying mechanisms and clinical significance of azole resistance and trailing in C. tropicalis bloodstream isolates are worth exploring further.

Supplementary Materials: The followings are available online at https:/ /www.mdpi.com/article/ 10.3390/jof7080612/s1, Figure S1: Minimum spanning tree of 64 C. tropicalis bloodstream isolates, Figure S2: Overview of the EUCAST microdilution ELISA read-outs of the twelve isolates in clonal complex 3, Figure S3: Scatter plot between geometric means of trailing percentages and MDR1 expression levels, Table S1: Primers for sequencing and quantitative RT-PCR.

Author Contributions: P.-Y.C. and Y.-C.C. (Yee-Chun Chen) conceived and designed the study. P.-Y.C., Y.-C.C. (Yu-Chung Chuang) and Y.-C.C. (Yee-Chun Chen) performed data analysis and interpretation. P.-Y.C. wrote the manuscript, which was reviewed and approved by Y.-C.C. (YuChung Chuang), U.-I.W., H.-Y.S., J.-T.W., W.-H.S. and S.-C.C., Y.-C.C. (Yee-Chun Chen) revised the article critically for important intellectual content and final approval of the version to be submitted. All authors have read and agreed to the published version of the manuscript.

Funding: This study was supported by the Ministry of Science and Technology (grant nos. 102-2314B-002-158-MY3, 104-2314-B-002-241 and 107-2314-B-002-181 to Y.-C.C., and grant no. 109-2314-B002-236-MY3 to P.-Y.C.), and the Ministry of Health and Welfare, Taiwan (grant nos. 107-TDU-B211-123002, 106-TDUB-211-113002 to Y.-C.C.). The funding source played no role in study design and conduct, data collection, analysis or interpretation, writing of the manuscript, or the decision to submit it for publication. 
Institutional Review Board Statement: The study was conducted according to the guidelines of the Declaration of Helsinki, and received National Taiwan University Hospital Institutional ReviewBoard approval (Study ID: 201502034RIND approved on 8 April 2015; Study ID: 201805097RIND approved on 6 June 2018).

Informed Consent Statement: The informed consent process was waived by the ethics committee.

Data Availability Statement: All data are available within the article and Supplementary Materials.

Acknowledgments: We thank Li-Fang Chen and Ching-Tzu Dai for laboratory support.

Conflicts of Interest: The authors declare no conflict of interest.

\section{References}

1. Pappas, P.G.; Lionakis, M.S.; Arendrup, M.C.; Ostrosky-Zeichner, L.; Kullberg, B.J. Invasive candidiasis. Nat. Rev. Dis. Primers 2018, 4, 18026. [CrossRef]

2. Tan, B.H.; Chakrabarti, A.; Li, R.Y.; Patel, A.K.; Watcharananan, S.P.; Liu, Z.; Chindamporn, A.; Tan, A.L.; Sun, P.L.; Wu, U.I.; et al. Incidence and species distribution of candidaemia in Asia: A laboratory-based surveillance study. Clin. Microbiol. Infect. 2015, 21, 946-953. [CrossRef] [PubMed]

3. Nucci, M.; Queiroz-Telles, F.; Alvarado-Matute, T.; Tiraboschi, I.N.; Cortes, J.; Zurita, J.; Guzman-Blanco, M.; Santolaya, M.E.; Thompson, L.; Sifuentes-Osornio, J.; et al. Epidemiology of candidemia in Latin America: A laboratory-based survey. PLoS ONE 2013, 8, e59373. [CrossRef]

4. Tan, T.Y.; Hsu, L.Y.; Alejandria, M.M.; Chaiwarith, R.; Chinniah, T.; Chayakulkeeree, M.; Choudhury, S.; Chen, Y.H.; Shin, J.H.; Kiratisin, P.; et al. Antifungal susceptibility of invasive Candida bloodstream isolates from the Asia-Pacific region. Med. Mycol. 2016, 54, 471-477. [CrossRef]

5. Wang, Y.; Shi, C.; Liu, J.Y.; Li, W.J.; Zhao, Y.; Xiang, M.J. Multilocus sequence typing of Candida tropicalis shows clonal cluster enrichment in azole-resistant isolates from patients in Shanghai, China. Infect. Genet. Evol. 2016, 44, 418-424. [CrossRef] [PubMed]

6. Xisto, M.I.; Caramalho, R.D.; Rocha, D.A.; Ferreira-Pereira, A.; Sartori, B.; Barreto-Bergter, E.; Junqueira, M.L.; Lass-Florl, C.; Lackner, M. Pan-azole-resistant Candida tropicalis carrying homozygous erg11 mutations at position K143R: A new emerging superbug? J. Antimicrob. Chemother. 2017, 72, 988-992. [CrossRef]

7. Chen, P.Y.; Chuang, Y.C.; Wu, U.I.; Sun, H.Y.; Wang, J.T.; Sheng, W.H.; Lo, H.J.; Wang, H.Y.; Chen, Y.C.; Chang, S.C. Clonality of fluconazole-nonsusceptible Candida tropicalis in bloodstream infections, Taiwan, 2011-2017. Emerg. Infect. Dis. 2019, 25, $1660-1667$. [CrossRef]

8. Arastehfar, A.; Daneshnia, F.; Hafez, A.; Khodavaisy, S.; Najafzadeh, M.J.; Charsizadeh, A.; Zarrinfar, H.; Salehi, M.; Shahrabadi, Z.Z.; Sasani, E.; et al. Antifungal susceptibility, genotyping, resistance mechanism, and clinical profile of Candida tropicalis blood isolates. Med. Mycol. 2020, 58, 766-773. [CrossRef] [PubMed]

9. Arastehfar, A.; Hilmioglu-Polat, S.; Daneshnia, F.; Hafez, A.; Salehi, M.; Polat, F.; Yasar, M.; Arslan, N.; Hosbul, T.; Unal, N.; et al. Recent increase in the prevalence of fluconazole-non-susceptible Candida tropicalis blood isolates in Turkey: Clinical implication of azole-non-susceptible and fluconazole tolerant phenotypes and genotyping. Front. Microbiol. 2020, 11, 587278. [CrossRef]

10. Tulyaprawat, O.; Pharkjaksu, S.; Chongtrakool, P.; Ngamskulrungroj, P. An Association of an eBURST group with triazole resistance of Candida tropicalis blood isolates. Front. Microbiol. 2020, 11, 934. [CrossRef]

11. Wang, Q.; Tang, D.; Tang, K.; Guo, J.; Huang, Y.; Li, C. Multilocus Sequence Typing Reveals Clonality of FluconazoleNonsusceptible Candida tropicalis: A study from Wuhan to the global. Front. Microbiol. 2020, 11, 554249. [CrossRef]

12. Jiang, C.; Dong, D.; Yu, B.; Cai, G.; Wang, X.; Ji, Y.; Peng, Y. Mechanisms of azole resistance in 52 clinical isolates of Candida tropicalis in China. J. Antimicrob. Chemother. 2013, 68, 778-785. [CrossRef] [PubMed]

13. Jin, L.; Cao, Z.; Wang, Q.; Wang, Y.; Wang, X.; Chen, H.; Wang, H. MDR1 overexpression combined with ERG11 mutations induce high-level fluconazole resistance in Candida tropicalis clinical isolates. BMC Infect. Dis. 2018, 18, 162. [CrossRef] [PubMed]

14. Fan, X.; Xiao, M.; Zhang, D.; Huang, J.J.; Wang, H.; Hou, X.; Zhang, L.; Kong, F.; Chen, S.C.; Tong, Z.H.; et al. Molecular mechanisms of azole resistance in Candida tropicalis isolates causing invasive candidiasis in China. Clin. Microbiol. Infect. 2019, 25, 885-891. [CrossRef]

15. Teo, J.Q.; Lee, S.J.; Tan, A.L.; Lim, R.S.; Cai, Y.; Lim, T.P.; Kwa, A.L. Molecular mechanisms of azole resistance in Candida bloodstream isolates. BMC Infect. Dis. 2019, 19, 63. [CrossRef] [PubMed]

16. Choi, M.J.; Won, E.J.; Shin, J.H.; Kim, S.H.; Lee, W.G.; Kim, M.N.; Lee, K.; Shin, M.G.; Suh, S.P.; Ryang, D.W.; et al. Resistance mechanisms and clinical features of fluconazole-nonsusceptible Candida tropicalis isolates compared with fluconazole-lesssusceptible isolates. Antimicrob. Agents. Chemother. 2016, 60, 3653-3661. [CrossRef]

17. Munoz, J.F.; Gade, L.; Chow, N.A.; Loparev, V.N.; Juieng, P.; Berkow, E.L.; Farrer, R.A.; Litvintseva, A.P.; Cuomo, C.A. Genomic insights into multidrug-resistance, mating and virulence in Candida auris and related emerging species. Nat. Commun. 2018, 9 , 5346. [CrossRef]

18. Bhattacharya, S.; Sae-Tia, S.; Fries, B.C. Candidiasis and mechanisms of antifungal resistance. Antibiotics 2020, 9, 312. [CrossRef]

19. Lee, Y.; Puumala, E.; Robbins, N.; Cowen, L.E. Antifungal drug resistance: Molecular mechanisms in Candida albicans and beyond. Chem. Rev. 2021, 121, 3390-3411. [CrossRef] 
20. Marcos-Zambrano, L.J.; Escribano, P.; Sanchez-Carrillo, C.; Bouza, E.; Guinea, J. Scope and frequency of fluconazole trailing assessed using EUCAST in invasive Candida spp. isolates. Med. Mycol. 2016, 54, 733-739. [CrossRef]

21. Marcos-Zambrano, L.J.; Gomez, A.; Sanchez-Carrillo, C.; Bouza, E.; Munoz, P.; Escribano, P.; Guinea, J. Isavuconazole is highly active in vitro against Candida species isolates but shows trailing effect. Clin. Microbiol. Infect. 2018, 24, 1343.e1-1343.e4. [CrossRef] [PubMed]

22. Astvad, K.M.T.; Sanglard, D.; Delarze, E.; Hare, R.K.; Arendrup, M.C. Implications of the EUCAST trailing phenomenon in Candida tropicalis for the in vivo susceptibility in invertebrate and murine models. Antimicrob. Agents Chemother. 2018, 62, e01624-18. [CrossRef] [PubMed]

23. Arendrup, M.C.; Meletiadis, J.; Mouton, J.W.; Lagrou, K.; Hamal, P.; Guinea, J. Subcommittee on antifungal susceptibility testing (AFST) of the ESCMID European Committee for Antimicrobial Susceptibility Testing (EUCAST). In EUCAST Technical Note on Method for the Determination of Broth Dilution Minimum Inhibitory Concentrations of Antifungal Agents for Yeast-EUCAST Definitive document E.DEF 7.3.1; EUCAST: Växjö, Sweden, 2017.

24. Marr, K.A.; Rustad, T.R.; Rex, J.H.; White, T.C. The trailing end point phenotype in antifungal susceptibility testing is pH dependent. Antimicrob. Agents Chemother. 1999, 43, 1383-1386. [CrossRef] [PubMed]

25. Lee, M.K.; Williams, L.E.; Warnock, D.W.; Arthington-Skaggs, B.A. Drug resistance genes and trailing growth in Candida albicans isolates. J. Antimicrob. Chemother. 2004, 53, 217-224. [CrossRef] [PubMed]

26. Jorgensen, K.M.; Astvad, K.M.T.; Hare, R.K.; Arendrup, M.C. EUCAST susceptibility testing of isavuconazole: MIC data for contemporary clinical mold and yeast isolates. Antimicrob. Agents Chemother. 2019, 63, e00073-19. [CrossRef]

27. Francisco, A.P.; Bugalho, M.; Ramirez, M.; Carrico, J.A. Global optimal eBURST analysis of multilocus typing data using a graphic matroid approach. BMC Bioinform. 2009, 10, 152. [CrossRef]

28. Vandeputte, P.; Larcher, G.; Berges, T.; Renier, G.; Chabasse, D.; Bouchara, J.P. Mechanisms of azole resistance in a clinical isolate of Candida tropicalis. Antimicrob. Agents Chemother. 2005, 49, 4608-4615. [CrossRef]

29. Forastiero, A.; Mesa-Arango, A.C.; Alastruey-Izquierdo, A.; Alcazar-Fuoli, L.; Bernal-Martinez, L.; Pelaez, T.; Lopez, J.F.; Grimalt, J.O.; Gomez-Lopez, A.; Cuesta, I.; et al. Candida tropicalis antifungal cross-resistance is related to different azole target (Erg11p) modifications. Antimicrob Agents Chemother. 2013, 57, 4769-4781. [CrossRef]

30. Jiang, C.; Ni, Q.; Dong, D.; Zhang, L.; Li, Z.; Tian, Y.; Peng, Y. The Role of UPC2 Gene in Azole-Resistant Candida tropicalis. Mycopathologia 2016, 181, 833-838. [CrossRef]

31. Desnos-Ollivier, M.; Bretagne, S.; Boullie, A.; Gautier, C.; Dromer, F.; Lortholary, O.; French Mycoses Study Group. Isavuconazole MIC distribution of 29 yeast species responsible for invasive infections (2015-2017). Clin. Microbiol. Infect. 2019, 25, 634. [CrossRef]

32. Chindamporn, A.; Chakrabarti, A.; Li, R.; Sun, P.L.; Tan, B.H.; Chua, M.; Wahyuningsih, R.; Patel, A.; Liu, Z.; Chen, Y.C.; et al. Survey of laboratory practices for diagnosis of fungal infection in seven Asian countries: An Asia Fungal Working Group (AFWG) initiative. Med. Mycol. 2018, 56, 416-425. [CrossRef]

33. Rosenberg, A.; Ene, I.V.; Bibi, M.; Zakin, S.; Segal, E.S.; Ziv, N.; Dahan, A.M.; Colombo, A.L.; Bennett, R.J.; Berman, J. Antifungal tolerance is a subpopulation effect distinct from resistance and is associated with persistent candidemia. Nat. Commun. 2018, 9, 2470. [CrossRef]

34. Chou, H.H.; Lo, H.J.; Chen, K.W.; Liao, M.H.; Li, S.Y. Multilocus sequence typing of Candida tropicalis shows clonal cluster enriched in isolates with resistance or trailing growth of fluconazole. Diagn. Microbiol. Infect. Dis. 2007, 58, 427-433. [CrossRef]

35. Sanglard, D.; Coste, A.T. Activity of isavuconazole and other azoles against Candida clinical isolates and yeast model systems with known azole resistance mechanisms. Antimicrob. Agents Chemother. 2016, 60, 229-238. [CrossRef] [PubMed]

36. Binder, U.; Aigner, M.; Risslegger, B.; Hortnagl, C.; Lass-Florl, C.; Lackner, M. Minimal Inhibitory Concentration (MIC)-phenomena in Candida albicans and their impact on the diagnosis of antifungal resistance. J. Fungi 2019, 5, 83. [CrossRef]

37. Levinson, T.; Dahan, A.; Novikov, A.; Paran, Y.; Berman, J.; Ben-Ami, R. Impact of tolerance to fluconazole on treatment response in Candida albicans bloodstream infection. Mycoses 2021, 64, 78-85. [CrossRef] [PubMed]

38. Rueda, C.; Puig-Asensio, M.; Guinea, J.; Almirante, B.; Cuenca-Estrella, M.; Zaragoza, O. Evaluation of the possible influence of trailing and paradoxical effects on the clinical outcome of patients with candidemia-For the members of the CANDIPOP project from GEIH-GEMICOMED and Reipi. Clin. Microbiol. Infect. 2017, 23, 49.E1-49.E8. [CrossRef]

39. Bhattacharya, S.; Sobel, J.D.; White, T.C. A Combination fluorescence assay demonstrates increased efflux pump activity as a resistance mechanism in azole-resistant vaginal Candida albicans isolates. Antimicrob. Agents Chemother. 2016, 60, 5858-5866. [CrossRef] [PubMed]

40. Arastehfar, A.; Lass-Florl, C.; Garcia-Rubio, R.; Daneshnia, F.; Ilkit, M.; Boekhout, T.; Gabaldon, T.; Perlin, D.S. The Quiet and Underappreciated Rise of Drug-Resistant Invasive Fungal Pathogens. J. Fungi 2020, 6, 138. [CrossRef] 